Jean Foyer* y David Dumoulin Kervran**

\title{
¿AMBIENTALISMO DE LAS ONG VERSUS AMBIENTALISMO DE LOS POBRES?
}

A principios de los 90, enganchados en la nueva ola de movilización que siguió a la democratización de América Latina, todos los países de la región parecían experimentar algún tipo de movimiento ambiental y la creación de agencias nacionales de medio ambiente (García-Guadilla y Blauert, 1994; Hochsteler y Mumme, 1998; Alfie Cohen, 2005a). La lucha ambiental, junto con las luchas por los derechos indígenas $\mathrm{y}$ los derechos de las mujeres, a menudo ha sido llamada "nuevo movimiento social (NMS)", que se distingue de los movimientos sociales anteriores por el estatus social más alto de sus miembros, por sus causas pos materialistas, basadas en la identidad y por la poca prioridad que le dieron a la acción directa realizada por medios extra institucionales (Melucci, 1999). Mientras que esta etiqueta de "NMS" tuvo todas las oportunidades para resaltar la aparición de nuevas formas de acción colectiva, también destacó la dimensión elitista ${ }^{1}$ de las mo-

* Institut des Sciences de la Communication, CNRS-Sorbonne Université-UPMC, París, Francia.

** Institut des Hautes Etudes de l'Amérique Latine (IHEAL) - CREDA, Université Sorbonne Nouvelle, París, Francia.

1 Este elitismo fue reforzado por el rol específico desempeñado por académicos y por la "política de información": el uso de datos científicos fue excepcionalmente intenso en las movilizaciones ambientales. 
vilizaciones ambientales y el papel conferido a las ONG. Por lo tanto, en México, la existencia de un movimiento social ambiental nacional parecía autoevidente para los escasos investigadores que lo adoptaron como tema específico (Simonian, 1999; Diez, 2008; Velázquez García, 2010; Durand et al., 2012) y su atención siguió centrada en las ONG. De hecho, aún hoy, la existencia y demarcaciones de este "movimiento social" siguen siendo muy controversiales entre sus grupos de interés, y cada investigador/a del medio ambiente debe indagar sobre sus propias definiciones.

Esta amplia dicotomía, entre los NMS más elitistas y las movilizaciones de base más populares, está arraigada en los dos marcos sociológicos principales que se utilizan actualmente en el análisis de la movilización ambiental en América Latina. Por un lado, muchas publicaciones enfatizan el papel de las ONG y coaliciones transnacionales, muy cercano al discurso de los actores más visibles (Kurzinger et al. 1991; Torres, 1997; Umlas, 1998; Hogenboom, 1998; Alfie Cohen, 2005b; Pacheco-Vega, 2005). Por otro lado, se otorga un papel más significativo a las organizaciones de base de las poblaciones marginadas y a las estrategias de confrontación, a través de marcos analíticos como el "ecologismo de los pobres" de Martínez Alier (2002), o el "movimiento de justicia ambiental" (Carruthers, 2008; Leff, 2001; Verduzco, 2002). Este último enfoque hace hincapié en los conflictos ambientales y en los movimientos indígenas y/o campesinos locales que se oponen a los proyectos de construcción de infraestructura (hidráulicos, mineros, nucleares, petroleros, turísticos, etcétera).

Sin embargo, esta vasta oposición entre los programas de investigación sobre las actividades de las ONG, por un lado, y las movilizaciones populares, por el otro, fue superado ampliamente durante los 90 (Clarke, 1995). Surgió una convergencia entre el estudio de la internacionalización de los movimientos sociales (Smith et al., 1998) y el papel de las ONG en las "redes activistas transnacionales" (Keck y Sikkink, 1998). Este nuevo marco analítico, que abarca tanto los movimientos sociales como las ONG, estableció una tipología evolutiva: la formación de coaliciones de articulación de actores heterogéneos a través de campañas constituyó un terreno intermedio entre una mera red de intercambio de información, por un lado, y la existencia de genuinos movimientos sociales transnacionales basados en identidad y estrategia compartidas, por el otro (Tarrow, 2005; Bandy y Smith, 2005).

En este capítulo, entonces, se basa en el análisis de las movilizaciones que se han centrado en la pluralidad de redes entre organizaciones (Diani y McAdam, 2003), un enfoque que solo se ha 
aplicado a determinados aspectos del movimiento mexicano (Pacheco-Vega y Obdulia, 2003; Velázquez García, 2008). A diferencia del análisis un tanto idealizado de los movimientos sociales enfocado exclusivamente en las movilizaciones populares de base, un enfoque centrado en las coaliciones puede mostrar que los diferentes actores movilizados están conectados entre sí tanto por la complementariedad y la división del trabajo, como por un conjunto compartido de valores comunes (Pacheco-Vega y Obdulia, 2003). El ambientalismo se caracteriza, con frecuencia, por la heterogeneidad "transclasista" de los participantes y, por lo tanto, la construcción de coaliciones es la forma más usual para ampliar la movilización. Este es el nexo distintivo entre las organizaciones "elitistas" y "populares", así como las dos trayectorias históricas distintas de alianzas, que nos permiten dividir empíricamente en dos componentes lo que podríamos llamar "ambientalismo social mexicano". ${ }^{2}$ En cambio, no vamos a tratar aquí con otro campo organizacional, el "conservacionismo", que reúne a las (numerosas) organizaciones que solo trabajan en la gestión de áreas protegidas (Dumoulin, 2003, 2007). No porque demos por sentado que las demandas de muchos conservacionistas sean apolíticas, sino porque sus actividades se centran demasiado en la "gestión de proyectos" para encajar en alguna definición de movimiento social.

Este estudio se basa en varios períodos de trabajo de campo realizado por los autores en México entre los años 2000 y 2012. ${ }^{3}$ Se fundamenta en más de 100 entrevistas con participantes de las organizaciones mencionadas, así como en períodos seleccionados de observación participante. Cada una de las dos partes del capítulo, entonces, explora la construcción de un "campo organizacional" basado en coaliciones entre las poblaciones locales, las élites urbanas y las organizaciones internacionales: llamamos a la primera parte "desarrollo comunitario sustentable" y la segunda "resistencia ambiental"; en la conclusión, vamos a volver a revisitar las principales ventajas de nuestra manera de construir el objeto "movimiento social ambientalista".

2 El término "ambientalismo social" no es comúnmente utilizado por los miembros de las organizaciones analizadas aquí. Esta categoría de análisis fue utilizada por González Martínez (1992) por primera vez.

3 Estos períodos de trabajo de campo tuvieron duraciones muy diferentes: de 2 años (durante la preparación de la tesis doctoral de cada uno de los autores) a numerosos periodos de 1 mes, durante la última década, dedicados a diferentes proyectos de investigación con temáticas relacionadas. 


\section{LAS REDES DEL DESARROLLO COMUNITARIO SUSTENTABLE: DESDE LAS BASES AL ESTADO Y LAS ENTIDADES DE FINANCIACIÓN}

\section{A. EL ORIGEN DE LAS ALIANZAS ENTRE LAS ONG Y LAS COMUNIDADES RURALES}

El primer paso en el establecimiento del campo gravitó en grupos urbanos, que a menudo se organizaban en grupos de la sociedad civil al forjar alianzas con las comunidades locales. En la segunda mitad de los años 70 y durante los 80, en México surgió una corriente ambientalista que estaba profundamente arraigada en el sector rural, y cuyas actividades principales se basaron más en la búsqueda del desarrollo comunitario alternativo que en la conservación de la naturaleza, que la distingue claramente de los conservacionistas tradicionales (Carruthers, 1996). En 1970, varios grupos de estudiantes decidieron tomar en serio la crisis ambiental y buscar alternativas en las zonas rurales. Reconocieron la influencia de diferentes escuelas de pensamiento, en general procedentes del marxismo y las repercusiones de la represión de 1968 en la academia, pero también de pensadores como Ignacy Sachs e Ivan Illich (que dirigió el Centro Intercultural de Documentación en México, entre 1961 y 1976). Otra figura inspiradora fue la del ingeniero agrónomo mexicano Efraím Hernández Xolocotzi, quien abogó por una "ciencia de huarache"4 (o ciencia en sandalias), es decir, una ciencia basada en el trabajo de campo, en contacto directo con la población rural y que incluye el reconocimiento de saberes y prácticas ancestrales. Después de crear los primeros centros autónomos de investigación ecológica, ${ }^{5}$ varios grupos personificaron el crecimiento de un movimiento orientado hacia el desarrollo comunitario sustentable.

Los miembros fundadores del Grupo de Estudios Ambientales (GEA), primero experimentaron durante varios años con nuevos programas de investigación, capacitando a las poblaciones locales y orquestando proyectos de desarrollo a nivel comunitario (forestales, del cultivo tradicional de maíz, etc.), antes de establecer formalmente su ONG en 1977 e implementar los proyectos alternativos en las comunidades rurales marginadas en varias regiones (Xalapa, Puebla, Guerrero, etcétera). Un segundo gran grupo estaba conformado por investigadores de la Universidad Nacional Autónoma de México

4 [N. de las T.] En español en el original.

5 Junto con el Centro de Ecodesarrollo, creado en 1972, y el Instituto de Ecología (1974), encontramos el Instituto Nacional de Investigaciones sobre Recursos Bióticos (INIREB), que encarnó el movimiento de "ambientalismo social" (1975-1988). Los tres seguían siendo muy dependientes del Estado mexicano. 
(UNAM), esencialmente biólogos, reunidos en torno del etnobotánico Víctor Manuel Toledo y la bióloga Julia Carabias, que estaban desplegando la oposición de izquierda al Partido Revolucionario Institucional (PRI). ${ }^{6}$ Gracias al éxito del primer experimento local de uno de los modelos de desarrollo alternativo, su grupo cambió la escala de los proyectos a través del Programa de Aprovechamiento Integral de Recursos Naturales (PAIR), que se extendió por las distintas regiones del país (Michoacán, Oaxaca y Durango) (Carabias et al., 1994; Toledo, 1983). Un tercer grupo de académicos activistas participó, durante la segunda mitad de los 80 , en el movimiento de las comunidades de la Sierra de Juárez del estado de Oaxaca para recuperar sus derechos territoriales sobre los bosques de las concesiones a empresas paraestatales. Fundaron la organización de Estudios Rurales y Asesoría (ERA). Esta primera movilización fue parte del nacimiento de uno de los movimientos más interesantes de la silvicultura comunitaria, cuando una organización local, la Unión Zapoteca-Chinanteca (UZA$\mathrm{CHI}$ ), fue entrenada en el uso sustentable de los recursos del bosque. De esta manera, los dos grandes sectores -la silvicultura comunitaria (Barton Bray y Merino Pérez, 2004) y la producción de café orgánico ${ }^{7}$ (Ejea y Hernández, 1991) - forjaron intensas relaciones entre los partidarios de las ONG y las organizaciones comunitarias, transformando los modos de desarrollo y los sistemas políticos locales por igual. La lucha por el control de los recursos naturales y la tierra -ya sea contra el Estado o contra los jefes locales- y los procesos de organización de las comunidades en sindicatos o cooperativas fueron fundamentales en esta primera fase.

Así, el movimiento ambiental sirvió como un punto de unión entre una elite urbana altamente politizada, proveniente del mundo académico o religioso, por un lado, y las comunidades locales que participaban en las luchas, por el otro. Esta alianza llevó, algunas veces, a relaciones de largo plazo entre las comunidades. La idea de

6 El Partido Socialista Unificado de México (PSUM) surgió de la fusión entre el Partido Comunista mexicano y varias corrientes de la izquierda mexicana. Ganó las elecciones municipales en la ciudad de Alcozauca, en el estado de Guerrero a finales de los 70. Esta victoria le dio al grupo la oportunidad de pasar de la reflexión a la acción política e implementar un proyecto experimental, que fue visto como una experiencia pionera del desarrollo sustentable en la región (principalmente, diagnóstico ambiental y un plan de gestión municipal).

7 Los ejemplos más representativos de esta ola de exportación orientada alrededor de las cooperativas de café orgánico fueron la Unión de Comunidades Indígenas de la Región del Istmo (UCIRI) y los Indígenas de la Sierra Madre de Motozintla (ISMAM). En estas organizaciones, los miembros de los grupos del tipo teología de la liberación fueron más influyentes que los miembros de la academia. 
que existía un movimiento indígena ambientalista que estaba anclado localmente en torno a las prácticas tradicionales (agua, suelo y manejo forestal) o en modos de producción alternativos (de café, miel, vainilla y así sucesivamente) fue desplegada por el movimiento de intelectuales, entre ellos Víctor Manuel Toledo, que llegó a evocar la idea seductora de "zapatistas verdes" (Toledo, 1992, 2000).

\section{B. INSTITUCIONALIZACIÓN Y RELACIONES CON EL ESTADO}

A principios de los 90, tras la Cumbre de la Tierra celebrada en Río de Janeiro en 1992, emergió una segunda generación de organizaciones alrededor del tema de desarrollo rural sustentable. Estas incluyeron, por citar solo algunos ejemplos destacados: el Grupo Interdisciplinario de Tecnología Rural Apropiada (GIRA), que operaba dentro de las comunidades indígenas de Michoacán; el Proyecto Sierra de Santa Marta (PSSM), en el sur del estado de Veracruz; y el Grupo GAIA, en la costa de Oaxaca. También se realizó un esfuerzo de coordinación a nivel nacional, a través del Consejo Civil Mexicano para la Silvicultura Sostenible (CCMSS), que se formó a mediados de los 90 para tratar de capitalizar las diferentes experiencias locales y promover la silvicultura comunitaria a nivel nacional, especialmente con las autoridades públicas.

Para entender el rápido desarrollo de las ONG ambientales mexicanas, debemos situarlo en el contexto nacional más amplio del proceso de democratización del sistema mexicano, que dejó espacio para ciertas experiencias fuera de corporativismo oficial (Mollard y López, 2006). Sin embargo, en un Estado nación que había sido controlado por el PRI durante casi 70 años, ${ }^{8}$ y un partido que se basaba en el corporativismo y el clientelismo, la cuestión de si forjar o no una relación con las instituciones oficiales planteó un dilema fundamental a todos los movimientos sociales mexicanos. El dilema estaba entre mantener la independencia a riesgo de quedar fuera de toda influencia institucional y recursos, ${ }^{9}$ o mantener el acceso a puestos de trabajo y beneficios (financieros $\mathrm{u}$ otros) a un riesgo aún mayor, el de legitimar un sistema establecido por el PRI y padecer bajo sus muchas limitaciones.

8 El PRI controló México desde el final de la Revolución en los años 20 hasta el año 2000. Después de una transición de 12 años dominada por la presidencia del derechista Partido de Acción Nacional (PAN), el PRI volvió al poder en 2012, a nivel nacional y en muchos estados.

9 De hecho, un estudio de 1991 (Kurzinger, 1991) mostró que el 75\% de las organizaciones consideradas tenía alguna relación con el Estado y que el 30\% recibió financiamiento estatal. 
Desde mediados de los 90, el grupo que se había formado alrededor de Julia Carabias y que conformaba PAIR tomó la decisión de institucionalizarse porque cuando Ernesto Zedillo llegó al poder, en 1994, se presentó la oportunidad de crear el primer Ministerio de Medio Ambiente mexicano, Secretaría del Medio Ambiente y Recursos Naturales (SEMARNAT).$^{10}$ Esta oportunidad también tuvo un correlato con el reverdecimiento del Gobierno mexicano a finales de los 80 en respuesta a la presión externa (las negociaciones del Tratado de Libre Comercio con América del Norte, TLCAN; la entrada en la Organización para la Cooperación y el Desarrollo Económico, OCDE) y la presión interna, incluyendo la canalización de muchos movimientos ambientales y su aparición durante ese momento como fuerza disidente (ver Parte II).

Por lo tanto, la integración de todo el equipo de Carabias al Estado también puede ser leída como parte de la gran tradición mexicana de cooptación de los movimientos sociales. Después de apropiarse del concepto de desarrollo sustentable, este equipo desempeñó seis años de ardua labor para consolidar el marco institucional y jurídico ambientalista oficial mexicano, para capacitar al personal administrativo en temas ambientales y para aumentar y fortalecer la supervisión de las áreas naturales protegidas, al tiempo que se tenían en consideración las actividades humanas. ${ }^{11}$

A pesar de las críticas extremadamente virulentas contra Carabias de parte de diversos movimientos sociales, desde su abordaje que se consideró excesivamente conservacionista a sus posturas contra el movimiento neozapatista en Chiapas, el sexenio de Julia Carabias al mando del Ministerio tuvo logros cuantitativos y cualitativos sin precedentes respecto del abordaje de las principales cuestiones ambientales en México. Este pasaje de las ONG a las instituciones y del ámbito local al nacional, a menudo implica un riesgo peligroso. Si los presupuestos que se manejan y los instrumentos implementados no tienen parangón con los de la sociedad civil, sus medios son insignificantes en comparación con otras políticas públicas, como las agropecuarias, y más estructurales y depredadores a nivel ambiental. Un miembro de GEA explicó las dificultades de traducir las iniciativas civiles en políticas públicas, de esta forma: "Si no entras

10 La Secretaría de Medio Ambiente, Recursos Naturales y Pesca que, habiéndose reducido del Sector Pesquero a finales de los 60, con J. Carabias al frente del Ministerio, se convirtió en la Secretaría de Medio Ambiente y Recursos Naturales (SEMARNAT). Ver Stearns y Almeida (2004), para los mutuos beneficios edificantes de las coaliciones de los movimientos sociales con las instituciones del Estado.

11 Julia Carabias, entrevista personal, 14 de octubre de 2004. 
en él, si no negocias, te marginan, y es importante posicionarse políticamente, incluso cuando podamos no estar vinculados a ningún poder del Estado o Partido". ${ }^{2}$

Otras organizaciones de campo que optaron por no asociarse con las autoridades estatales mantuvieron una sutil posición contra el Estado: más allá de algún discurso crítico que podría implicar un mayor rechazo del Estado corporativo, parece haber sido aceptada cierta flexibilidad por medio de las colaboraciones con las instituciones ambientales. A criterio de los cambios de personal en las oficinas centrales de la SEMARNAT, así como en los diferentes estados, las relaciones con los miembros de las agencias de gobierno, los nuevos programas y las oportunidades de financiamiento, eventualmente abrieron grietas y "ventanas de oportunidad" a los miembros del movimiento para el desarrollo comunitario sustentable. En relación a las instituciones nacionales e internacionales, pareció prevalecer algún grado de pragmatismo.

\section{LAS CONEXIONES DE LA GLOBALIZACIÓN: EL FINANCIAMIENTO YLA PROFESIONALIZACIÓN}

Al desempeñar un papel acaso marginal aunque sin dudas pionero, las organizaciones del ambientalismo social estaban bien posicionadas para recibir, a partir de 1992, el flujo del financiamiento internacional para cuestiones de desarrollo y biodiversidad sustentables. ${ }^{13}$ Hay que destacar cierto grado de similitud en los canales de asociación y financiación internacionales, pues ilustra la existencia de las mismas redes transnacionales (la Fundación Ford, las agencias de cooperación del norte de Europa y el Banco Mundial) ${ }^{14}$ muy dis-

12 [N. de las T.] De la traducción original al inglés de L. Kraftowitz.

13 Durante los años 80, la mayoría de estas organizaciones se autofinanciaba; muy raramente estaban financiadas con fondos públicos.

$14 \mathrm{La}$ más visible es la Fundación Ford (junto a las fundaciones Rockefeller, McArthur y la alemana Friedrich Ebert) que casi siempre estaba presente apoyando a estas organizaciones, al menos hasta fines de los 90. Además, Oxfam Novib (la organización holandesa de cooperación internacional para el desarrollo) así como las organizaciones religiosas alemanas Misereor y Brot für die Welt (Pan para el Mundo), y las agencias de cooperación del norte de Europa (Escandinavia y Alemania, pero también el Reino Unido a través de DFID y el Departamento para el Desarrollo Internacional). El fondo administrado por el Banco Mundial, General Environmental Facility (GEF), también fue una fuente de financiación importante permitiendo a estos actores consolidar o crear nuevas organizaciones. Con respecto a las múltiples relaciones existentes entre estas ONG y el Banco Mundial ver, por ejemplo, Deborah A. Bräutigam y Monique Segarra (2007). 
tintos de los de la resistencia ambiental. Sin embargo, nos podemos preguntar si la dependencia estructural vis a vis los donantes institucionales internacionales (así como los fondos nacionales, tales como los distribuidos por el Fondo Mexicano para la Conservación de la Naturaleza) no ayudó a socavar los aspectos alternativos y activistas de las organizaciones de desarrollo comunitario, transformándolas en proveedoras de servicios y operadoras técnicas locales.

Desde esta perspectiva, el ejemplo de PSSM es significativo. La organización finalmente demostró una estrecha relación con las diferentes instituciones de la Reserva de la Biosfera de Los Tuxtlas, ya sea a través de la participación en su diseño ecológico, la programación, los órganos de gobierno y la gestión de algunos de sus programas; lo que nos lleva legítimamente a suponer si no se convirtió en una parte del sistema oficial. En un contexto ambiental nacional e internacional donde se han institucionalizado las cuestiones ambientales, la elección de los temas a trabajar, en parte, está determinada por las oportunidades de financiación. Luisa Paré, la fundadora de la organización y activista ambiental por más de 20 años, expuso el siguiente análisis:

Pasamos de una época de activismo con un fuerte compromiso político por parte de quienes vivieron y trabajaron en las comunidades, a tener hoy una actitud más profesional, donde las personas están realmente tratando de cambiar las cosas y de priorizar los aspectos técnicos de su trabajo. No estoy juzgando, simplemente estoy diciendo que estos son otros tiempos y otras formas de acción. ${ }^{15}$

Por tanto, la llegada de fondos extranjeros permitió cierto grado de profesionalización organizacional, así como la adopción de líneas de trabajo que, aunque seguían relacionadas con la promoción de proyectos de desarrollo local, estuvieron cada vez más institucionalizadas y restringidas al desarrollo sustentable. En la década de los 90 y 2000, el desarrollo comunitario estaba cada vez más inserto en redes gestionadas y en el financiamiento institucional nacional e internacional. En gran parte, la alternativa social y productiva que podría haber representado el desarrollo rural sustentable fue estandarizado amplia y gradualmente a medida que el desarrollo sustentable llegó a dominar las políticas nacionales e internacionales (Leonard y Foyer, 2011) (ver Cuadro 16.1).

15 Entrevista personal, 3 de octubre de 2008. 


\section{Cuadro 16.1}

La construcción de un "campo organizacional" denso para el "desarrollo comunitario sustentable"

\begin{tabular}{|c|c|c|c|}
\hline & $\begin{array}{l}\text { Escala de las movilizaciones } \\
\text { de redes sociales }\end{array}$ & $\begin{array}{l}\text { Tipos de vínculos } \\
\text { y coaliciones }\end{array}$ & Ejemplos \\
\hline$A$ & $\begin{array}{l}\text { Conexiones entre ONG } \\
\text { y comunidades locales }\end{array}$ & $\begin{array}{l}\text { Vínculos de apoyo técnico de } \\
\text { largo plazo; respaldo político, } \\
\text { financiero y representativo }\end{array}$ & $\begin{array}{l}\text { Relaciones entre CCMSS y } \\
\text { las comunidades de la selva } \\
\text { en Sierra Norte de Oaxaca }\end{array}$ \\
\hline B & $\begin{array}{l}\text { Conexiones entre ONG, } \\
\text { organizaciones locales y } \\
\text { distintos áreas de gobierno }\end{array}$ & $\begin{array}{l}\text { Nueva relación desde } \\
\text { 1994; influencia mutua e } \\
\text { interdependencia financiera. } \\
\text { Viejas redes sociales } \\
\text { entre nuevas entidades } \\
\text { ambientales, ONG, etcétera }\end{array}$ & $\begin{array}{l}\text { Las relaciones con los } \\
\text { gestores del programa } \\
\text { PRODERS en los estados, y } \\
\text { los del FMCN en México D.F. }\end{array}$ \\
\hline C & $\begin{array}{l}\text { Conexiones transescalares } \\
\text { con donantes } \\
\text { internacionales }\end{array}$ & $\begin{array}{l}\text { Dependencia financiera, } \\
\text { legitimidad reciproca }\end{array}$ & $\begin{array}{l}\text { Relaciones con el Banco } \\
\text { Mundial, el PNUD, las } \\
\text { Fundaciones Ford } 0 \\
\text { Friedrich Ebert. }\end{array}$ \\
\hline
\end{tabular}

\section{RESISTENCIA AMBIENTAL: DE LAS COALICIONES CONTRA LOS PROYECTOS LOCALES A LAS COALICIONES CONTRA EL ORDEN NEOLIBERAL}

\section{A. COALICIONES DE RESISTENCIA A LOS MEGAPROYECTOS Y COORDINACIÓN NACIONAL}

Algunas campañas de resistencia contra el desarrollo de megaproyectos, en paralelo y a veces transversales a los campos organizacionales de desarrollo comunitario, también crearon sinergia entre algunas elites urbanas y organizaciones de base locales. Entre finales de los 70 y principios de los 80 , cuando los ecologistas todavía eran poco frecuentes, dos coaliciones dieron visibilidad a un incipiente movimiento ambiental mexicano que confrontó al Estado: (1) la lucha contra los planes de deforestación y (2) la reubicación de los Chinantecos de Uxpanapa (Veracruz 1973-1975). El surgimiento de las movilizaciones contra la perforación petrolífera y la contaminación masiva del gigante petrolero nacional de PEMEX estuvo aún más conectado con las organizaciones populistas, especialmente el Pacto Ribereño de 1976 en el estado de Tabasco (Velázquez Guzmán, 1982).

La movilización antinuclear de 1987, la Coordinadora Nacional contra Laguna Verde (CONCLAVE), reunió a grandes sectores de la población: organizaciones de agricultores y pescadores, ONG, intelectuales, grupos de madres y hasta la Iglesia Católica (García-Gorena, 1999; Payá Porres, 1994). Esta movilización experimentó episodios 
de confrontación radical (corte de calles, etc.) y es considerada como la fundadora del movimiento. Los años 90 fueron un teatro de operaciones para grandes protestas cuyos éxitos ilustran la capacidad de las ONG para participar directamente en el trabajo político con las "organizaciones de base" en las regiones marginadas y el papel central que podrían desempeñar en la movilización de las coaliciones desde un encuadre ambientalista (Verduzco, 2002). Esto fue cierto, especialmente, para las movilizaciones contra los proyectos de represas (en Guerrero contra "Altos Balsas", a partir de 1990), contra el proyecto de carretera en la región de los Chimalapas en 1991 (Umlas, 1998) y contra de la construcción de una fábrica de sal en la laguna San Ignacio en Baja California (1995-2000) (Castro-Soto, 2005; Velásquez García, 2010).

En algunos casos, como con la famosa victoria contra el proyecto del complejo turístico de Tepoztlán (1994-1995), las organizaciones campesinas condujeron luchas donde el medioambiente fue solo un elemento entre otros. Por el contrario, algunas coaliciones tuvieron objetivos más "naturalistas" y limitados, ligados a varias ONG en el ámbito nacional, como la demanda de responsabilidad por el sacrificio de las aves migratorias en la represa Silva en 1994-1995. Estas diversas experiencias conectaron a un número creciente de organizaciones locales dispersas en varios estados en una coalición ad hoc junto con las ONG que participaban en el escenario nacional, y los muchos éxitos contribuyeron a crear recuerdos y lazos sociales compartidos. Sin embargo, casi no hubo una coordinación general entre estos "eventos de protesta", excepto a través de las redes sociales informales preexistentes.

Desde 1985 hasta 1994, numerosos grupos de ecología política urbana en un estado de rápido crecimiento intentaron organizar un movimiento nacional, para crear una identidad común bajo la consigna de "política contenciosa". Varias iniciativas reunieron a diversas coaliciones, aunque sin poder unificarlas. Los encuentros nacionales de los grupos ecologistas, los intentos de unificar el movimiento ambiental en la Federación Conservacionista Mexicana (FECOMEX) en 1985, el mismo año en que el gran terremoto catalizó la sinergia organizacional intersectorial y que, además, generó el "Pacto de Grupos Ecologistas" (PGE, establecido entre 50 organizaciones que compartían una agenda nacional) fueron todos intentos de coordinar organizadamente un movimiento nacional. Las negociaciones de la ONU en la Conferencia de Río 92 generaron la creación de FOROMEX, que reúne a más de 100 organizaciones en torno a una agenda común (Diez, 2008: 86). El principal punto de inflexión fue, probablemente, cuando se formaron coaliciones alrededor del TLCAN entre $1990 \mathrm{y}$ 
1994. De hecho, estas iniciativas requieren que los grupos desarrollen una agenda común contra un enemigo común, y la ventana de oportunidad les dio un nuevo nivel de visibilidad e influencia en el Estado de México (Torres, 1997; Hogenboom, 1998; Gallardo, 1999).

Todas estas alianzas permitieron el surgimiento gradual de coaliciones y de una agenda multisectorial compartida ${ }^{16}$ en una nueva escala transnacional, que marcaron las protestas que siguieron. Pero la institucionalización de la causa ambiental también dividió y desestabilizó estas coaliciones, que ahora contienen una membresía muy diversa, con posiciones ideológicas muy arraigadas y sensibles que favorecen diferentes estrategias (Hogenboom, 1998; Hogenboom et al., 2003).

\section{B. TRANSNACIONALIZACIÓN DE LAS REDES Y RESISTENCIA ANTINEOLIBERAL}

Desde finales de los 90, la red de resistencia ambiental creció al estructurarse alrededor de temas antineoliberales a nivel nacional e internacional. Se acercó más a las movilizaciones campesinas e indígenas, especialmente las organizaciones cuya tarea es defender la agenda nacional, actores cercanos al neozapatismo y el movimiento antiglobalización. Así, a nivel nacional, se fue formando en México una organización importante que produciría conocimientos técnicos y políticos sobre temas ambientales en las zonas rurales. El Centro de Estudios para el Cambio en el Campo Mexicano (CECCAM), inicialmente, fue concebido como un foro de reflexión (think tank) para generar ideas políticas para la federación campesina, la Unión Nacional de Organizaciones Regionales Campesinas Autónomas (UNORCA), miembro de La Vía Campesina. El discurso de CECCAM, que cubría la silvicultura, la agroecología y la biotecnología, destacó la penetración gradual de las cuestiones ambientales en el mundo de las organizaciones rurales. Organizaciones como CECCAM, que tenían una perspectiva nacional, exhibieron cierta maduración social ambientalista, a medio camino entre la experiencia técnica y el activismo de RRPP, una posición que los acercó más a las organizaciones de expertos activistas y a las redes transnacionales de activismo. ${ }^{17}$

16 Sin embargo, tenemos que considerar que este programa estaba fuertemente influenciado por la agenda internacional, mucho más que por algunas características específicas de México (cf. Alfie Cohen, 1995).

17 Vale la pena recordar el levantamiento de uno de los primeros movimientos campesinos, que desde 1997 se autoidentificaron claramente como ecologistas. Sucedió en la Costa Grande del estado de Guerrero; Rodolfo Montiel y Teodoro Cabrera fueron sus dos líderes. Estuvieron presos desde 1999 hasta 2001, y luego 
Las organizaciones que se establecieron en Chiapas, como Maderas del Pueblo del Sureste y CIEPAC (Centro de Investigaciones Económicas y Políticas de Acción Comunitaria) denunciaron varias iniciativas ambientales del Gobierno mexicano, como la reubicación de las poblaciones locales fuera de la Reserva de la Biosfera de Montes Azules, sus proyectos de bioprospección como el ICBG-Maya (Dumoulin Kervan y Foyer, 2004), lujosos proyectos de ecoturismo e iniciativas como el Corredor Biológico Mesoamericano (CBM), que fue considerado como la parte ambiental de la iniciativa regional Puebla-Panamá. Inscribiéndose en el enfoque autonomista del zapatismo, en ruptura directa con el Gobierno, estas organizaciones se alejaron de las actividades de desarrollo comunitario y avanzaron con campañas de denuncia contra el ecologismo oficial al que consideraban demasiado conservacionista, si no al servicio de los intereses de las corporaciones multinacionales.

La segunda mitad de los 90 y principios de los 2000 se correspondió con la llegada de actores transnacionales que influyeron en el paisaje ya denso del ecologismo social mexicano. Catherine Marielle de la GEA resumió lo que considera es una nueva fase:

La llegada de Greenpeace y el Grupo ETC es mucho más reciente. A diferencia de estas organizaciones, no tenemos tiempo para trabajar en la visibilidad, verdaderamente, como lo hace Greenpeace, como una estrategia de recaudación de fondos. Esta es una fase muy distinta, y muy nueva, con sus propias características correspondientes a la globalización. Estas organizaciones llegaron después de Río 92. "Antes, nuestro trabajo estaba mucho más situado en el plano nacional, mientras que ahora estamos participando en redes internacionales". ${ }^{18}$

Aunque Greenpeace había estado presente en México desde 1993, con las campañas contra la contaminación del aire en la ciudad de México, contra la central nuclear de Laguna Verde y con campañas denunciando la importación de residuos tóxicos, no fue sino hasta 1998 que decidió iniciar en México una campaña de "ingeniería genética". México fue considerado como un área estratégica en este tema, debido a su biodiversidad agrícola y el riesgo planteado por las importaciones de Estados Unidos ${ }^{19}$ En 1999, Silvia Ribeiro, representante

fueron obligados a exiliarse. En el año 2000 ganaron el prestigioso Premio Ambiental Goldman por su trabajo.

18 Entrevista personal, 22 de enero de 2006. [N. de las T.] De la traducción original al inglés de L. Kraftowitz.

19 Uno de los objetivos era el de supervisar y fortalecer tanto como fuera posible la 
de RAFI (Rural Advancement Foundation International, que en 2001 cambió el nombre por ETC Group) y con experiencia en el periodismo de defensa de derechos, llegó a México desde Uruguay. A pesar de sus restricciones organizativas, Greenpeace y ETC Group desempeñaron un papel central en iniciar y orquestar las campañas contra la bioprospección y el maíz transgénico. Significativamente, introdujeron en México algunos de los principales métodos comunicativos y se convirtieron en puntos nodales en la formación de una incipiente red de oposición.

Prontamente crearon alianzas con las organizaciones ambientales y campesinas mexicanas. De esta manera, Greenpeace se vinculó con GEA y ANEC (otro gremio de La Vía Campesina) para fundar el movimiento "Sin Maíz, No hay País", mientras que ETC Group se acercó a CECCAM y UNORCA y formó la Red en Defensa del Maíz. En líneas generales, la llegada de estas organizaciones internacionales en el contexto de la globalización parece haber "traccionado" a una sección de actores del ecologismo social mexicano del ámbito local al global, y de problemas concretos arraigados en las comunidades locales a problemas más estructurales definidos en términos de política nacional e internacional. Por lo tanto, la confluencia de las movilizaciones ambientales en coaliciones multisectoriales representa el gran desafío de este nuevo período. Las movilizaciones durante la Cumbre de la OMC en Cancún en 2003 enfatizaron las divisiones y los riesgos del anhelo de algunas ONG de redes de coordinación adecuadas. Con este cambio en la escala de percepciones, una parte del ecologismo mexicano se sumó a la reconfiguración ideológica representada por la oposición a la globalización neoliberal. ${ }^{20}$ Es importante destacar que algunos personajes claves de este movimiento fueron incorporados al nuevo Ministerio de Medio Ambiente a cargo de Lichtinger en 2001, después de que el PRI fuera derrotado. Sin embargo, lejos de cumplir las expectativas, el Ministerio fue incapaz de impulsar una mayor justicia ambiental y, en cambio, perdió poder político.

En paralelo, en la década de 2000 también continuaron las movilizaciones contra los megaproyectos, marcadas por este carácter

moratoria del maíz transgénico, que había sido declarada por el gobierno mexicano en ese momento (Gustavo Ampugnani, entrevista personal, 23 de enero de 2006), otro era impedir la autorización de maíz transgénico mexicano, en particular a través de activismo y medidas legales.

20 Se puede observar esta reconfiguración ideológica a través de la denuncia sistemática del acuerdo comercial del TLCAN y por el fuerte apoyo dado al movimiento neozapatista, que constituye un punto de referencia principal del movimiento altermundialista. 
nuevo y radical de las organizaciones campesinas e indígenas, que ahora se integraron al debate medioambiental con un discurso político cada vez más estructurado en los ámbitos rurales y nacionales. Mientras algunas luchas violentas -como las movilizaciones contra el aeropuerto en San Salvador Atenco en 2001-2002 (Davis y Rosan, 2004) y contra la represa "La Parota" en el estado de Guerrero en 2003-2012 (Castro-Soto, 2005)- fueron exitosas, sobre todo fue una época donde los proyectos mineros a gran escala avanzaron muy rápido en vastas secciones del territorio nacional. Así, el dinamismo de la Asamblea Nacional de Afectados Ambientales (ANAA), que nació en 2008 de coaliciones y "caravanas" previas, ilustra la gravedad de las condiciones locales y el intento de una lucha ambiental unificada contra las políticas neoliberales (ver Cuadro 16.2).

\section{Cuadro 16.2}

Las cuatro vías para movilizar un repertorio de oposición radical en una amplia base social: de la acción colectiva minimalista transformadora a un fuerte movimiento social

\begin{tabular}{|c|c|c|}
\hline & $\begin{array}{l}\text { Intensificación de redes } \\
\text { de movilización social }\end{array}$ & Ejemplos \\
\hline A & $\begin{array}{l}\text { Acción colectiva basada en una conexión } \\
\text { local - nacional }\end{array}$ & $\begin{array}{l}\text { Consejo Mexicano para la Silvicultura } \\
\text { Sustentable (CMSS) y asociados }\end{array}$ \\
\hline B & $\begin{array}{l}\text { Coalición de numerosas organizaciones } \\
\text { ambientales nacionales }\end{array}$ & $\begin{array}{l}\text { Red Nacional de Derecho a la Información } \\
\text { Ambiental (RNDIA) en } 2001^{*} \text {, Presa Silva, } \\
\text { Foromex }\end{array}$ \\
\hline C & $\begin{array}{l}\text { Coaliciones amplias que conectan ONG } \\
\text { nacionales y transnacionales con una fuerte } \\
\text { movilización local contra grandes proyectos } \\
\text { de desarrollo }\end{array}$ & $\begin{array}{l}\text { Laguna Verde (1987-1988), Tepoztlán } \\
\text { (1995-1996), San Salvador Atenco (2002), } \\
\text { Presa La Parota (2003-2012) }\end{array}$ \\
\hline D & $\begin{array}{l}\text { Grandes coaliciones multisectoriales en las } \\
\text { cuales las organizaciones ambientales son } \\
\text { sólo un componente de una movilización } \\
\text { social más amplia }\end{array}$ & $\begin{array}{l}\text { Tepoztlán, "Sin Maïs no hay Païs”, } \\
\text { campañas, foros sociales. }\end{array}$ \\
\hline
\end{tabular}

Ref.: a. "Su creación se dio en el marco del I/ Encuentro Nacional de Ecologistas, donde participaron 50 organizaciones de 26 estados del país" (Ver: Velázquez, 2005).

\section{CONCLUSIÓN}

En el caso de México, la oposición entre un "ambientalismo de los pobres" con movimientos populares y comunidades locales movilizadas como base, por un lado, y una red de ONG transnacionales, por otro lado, no refleja la complejidad de los diferentes componentes del movimiento social ambiental. Basados en el análisis de estas diferentes organizaciones y sus trayectorias de más de 30 años, en este capítulo 
sostenemos que este movimiento se compone de dos "campos organizacionales" distintos. Cada uno se desarrolló a partir de una coalición diferente entre ONG y organizaciones locales, que luego formó parte de redes nacionales, internacionales o transnacionales más amplias. En estos dos campos, podemos identificar este tipo de "transclasismo", una característica típica de los movimientos ambientales, así como una extensión de las coaliciones de lo local a lo transnacional. Más allá de las estigmatizaciones agraviantes recíprocas de "elitismo" y "populismo", el mejor criterio para diferenciar estas dos formas de articulación entre organizaciones locales, regionales, nacionales y transnacionales debe ser el repertorio de acción colectiva. De hecho, las coaliciones de desarrollo comunitario sustentable son más "reformistas" y se centran en la construcción de alternativas para los más pobres, mientras que los movimientos de resistencia ambiental ponen en primer plano la "política contenciosa" en nombre de los dominados. ${ }^{21}$ Estos dos métodos de agregación no solo definen dos lados del movimiento social ambiental mexicano, sino que además coinciden con dos definiciones académicas distintas de "movimiento social". 22

Aunque "el desarrollo comunitario sustentable" parece encajar bastante bien con el diagnóstico de la oenegeización y tecnificación, el análisis histórico de este tipo particular de movilización demuestra que no se lo puede reducir a un proceso de este tipo. En términos generales, la tendencia hacia la "oenegeización" de los movimientos sociales por medio de la institucionalización no es unilateral (Alvarez, 1999; Diez, 2008). Algunas viejas organizaciones formales, de hecho, estaban desde antes de las movilizaciones más grandes y algunos ciclos de repolitización se han producido después de la tendencia a la institucionalización. Por otro lado, Alvarez (1999) identificó con precisión la "doble identidad" de los líderes que, a pesar de la institucionalización conservaron redes y repertorios mixtas entre las ONG y la dinámica del movimiento social (Velásquez, 2005). En primer lugar, este campo sin duda encuentra su origen en las movilizaciones colectivas, con una dimensión abiertamente política. De hecho, la oposición directa al Estado y la participación de la comunidad desempeñaron un papel crucial; y, hoy día, una parte importante de estas organizaciones todavía mantiene estrechos vínculos con el campo de la "resistencia ambiental". Por otra parte, las relaciones poderosas

21 Esta polaridad se aproxima a la propuesta por Pleyers (2010) en su análisis en profundidad del movimiento alter-mundialista: entre la experimentación y la experiencia alternativa.

22 Se puede citar a Touraine (1981) como ejemplo de la primera y a Tarrow (1998) de la segunda. 
que conectan a las ONG del desarrollo comunitario sustentable con las organizaciones locales en las regiones marginadas, que actualmente están más "profesionalizadas" siempre han tenido y mantienen un papel clave. En algunos casos, esto ha dado lugar a procesos de organización, autonomía y empoderamiento a largo plazo, y ha permitido la adopción de nuevas prácticas productivas (silvicultura comunitaria, cultivo de café orgánico, comercio justo...), que luego transformaron las relaciones políticas que tenían estas comunidades con actores externos. Por supuesto, la institucionalización de este componente es percibida, a veces, como la muerte de un movimiento ambiental que se basa en una política contenciosa. Pero, por otro lado, esta dinámica también puede ser vista como la mejor manera de ganar influencia sobre las instituciones y metanormas que regulan la relación que la sociedad mexicana tiene con su entorno. Esta producción de símbolos, información y leyes desempeña un papel de liderazgo en la transformación del modelo desarrollista (Azuela, 2006).

Si nos fijamos en sus estrategias de confrontación directa desplegadas por medios extra institucionales, así como en su discurso antisistema, más crítico y radical contra el Estado, el neoliberalismo y las empresas transnacionales, el campo de la "resistencia ambiental" parece encajar a la perfección en la definición más clásica de movimientos sociales. Sin embargo, en esta ocasión las cosas son más complejas, pues observamos fenómenos de institucionalización en algunas redes altermundistas y que algunas de estas afirmaciones universales a veces pueden ser consideradas elitistas, como cuando los temas más candentes de la agenda están más cerca de los reclamos de la población de alto nivel educativo, urbana y de "vanguardia" que de los reclamos indígenas o campesinos. Tanto la estructura de oportunidades políticas como la forma de las movilizaciones han experimentado una importante transformación desde 1990. Hay que destacar una doble tendencia: la transnacionalización de la mayoría de las redes y agendas, y al mismo tiempo, un retorno hacia las luchas políticas locales (Velázquez García, 2010; Pleyers, 2011). Las organizaciones ambientales de raigambre local han florecido en la última década, pero dado que carecen de visibilidad y que no buscan grandes acciones, el análisis en profundidad emergente de algunas de estas redes locales posee un gran valor (Velázquez, 2009, Lutz Ley y Salazar Adams, 2011).

Por último ¿quiénes son los verdaderos ambientalistas? ¿Aquellos que están construyendo alternativas de desarrollo con las comunidades marginadas, o los que participan en las luchas contra las manifestaciones más agresivas de un modelo destructivo? Esta polémica, que inquieta a los activistas y analistas por igual, es realmente inútil. 
Estos dos componentes se oponen al orden social dominante y constituyen las dos caras de lo que verdaderamente debe ser llamado "ambientalismo social mexicano".

\section{BIBLIOGRAFÍA}

Alfie Cohen, M. 1995 "La realidad del movimiento ecologista en México" en Desarrollo Sustentable, N 70: 14-18, julio-agosto.

Alfie Cohen, M. 2005a Democracia y desafío medioambiental en México. Retos, riesgos y opciones en la nueva era de la globalización (México-Barcelona: Pomares / UAM-A).

Alfie Cohen, M. 2005b "Auge y caída de las ONG ambientalistas" en Memorias (México: UAM-A).

Alvarez, S. 1999 'Advocating Feminism: The Latin American feminist NGO boom' en International Feminist, Journal of Politics, 1(2): 181-209.

Azuela, A. 2006 Visionarios y pragmáticos. Una aproximación sociológica al derecho ambiental (México: UNAM-IIS / Fontamara).

Bandy, J. y Smith, J. (ed.). 2005 Coalitions across borders. Transnational protest and the neoliberal order (Lanham: Rowman \& Littlefield).

Barton Bray, D. y Merino Pérez, L. 2004 La experiencia de las comunidades forestales en México (México: INE-SEMARNAT).

Carabias, J.; Provencio, E. y Toledo, C. 1994 Manejo de recursos naturales y pobreza rural (México: Fondo de Cultura Económica / UNAM).

Carruthers, D. V. 1996 'Indigenous ecology and the politics of linkage in Mexican social movements' en Third World Quaterly, 17(5): 1.007-1.028.

Carruthers, D. V. 2008 Environmental justice in Latin America: Problems, promise, and practice (Cambridge: MIT Press).

Castro Soto, G. 2005 "México: presa La Parota. Historia de un fraude" en Biodiversidad en América Latina, 13 de octubre. En $<$ www.biodiversidadla.org/content/view/full/19456> acceso 15 de julio de 2013.

Clarke, G. 1995 'Non-Governmental Organizations (NGO) and politics in the developing world' en Political Studies, 46: 36-52.

Davis, D. y Rosan, C. 2004. 'Social movements in the Mexico City airport controversy: Globalization, democracy and the power of distance' en Mobilization, 9(3): 279-293.

Diani, M. y McAdam, D. (eds.). 2003 Social movements and networks (Oxford University Press). 
Diez, J. 2008 'The rise and fall of Mexico's Green Movement' en European Review of Latin American and Caribbean Studies, 85: 81-99.

Dumoulin, D. 2003 'Local knowledge in the hands of transnational NGO networks: A Mexican viewpoint' en International Journal of Social Sciences, 178: 593-605.

Dumoulin Kervan, D. 2007 "Las políticas de las ANP (Areas Naturales Protegidas) como laboratorio para los esquemas público-privado. Una interpretación a partir del Fondo Mexicano para la Conservación de la Naturaleza" en Van Vliet, G. y Fontaine, G. (dir.) Viajes en los terruños de la gobernabilidad en las políticas ambientales en América Latina (Quito, FLACSOEcuador) pp. 57-78.

Dumoulin Kervan, D. y Foyer, J. 2004 "ONG transnationales et experts dans le débat démocratique: bioprospection et savoirs indigènes au Mexique" en Problèmes d'Amérique latine, 54: 95-122.

Figueroa, F. y Guzmán, M. (eds.). 2012 La naturaleza en contexto. Hacia una ecología política mexicana (México D. F.: UMANCRIM / El Colegio de San Luis A.C.).

Ejea, G. y Hernández, L. (eds.). 1991 Cafetaleros: la construcción de la autonomía (México D. F.: CNOC / Servicio de Apoyo Local).

Gallardo, S. C. 1999 Acción Colectiva y Diplomacia Social: Movimientos Ambientalistas frente al Tratado de Libre Comercio de América del Norte (México: El Colegio de México) Tesis doctoral.

García-Gorena, V. 1999 Mothers and the Mexican AntiNuclear Power Movement (Tucson: University of Arizona Press).

González Martínez, A. 1992 'Socio-ecological struggles in Mexico: The prospects' en International Journal of Sociology and Social Policy, 12(4-7): 113-128.

Garcia-Guadilla, M. P. y Blauert, J. (eds.) 1994 Retos para el desarrollo y la democracia: movimientos ambientales en América Latina y Europa (Caracas: Editorial Nueva Sociedad).

Hochsteler, K. y Mumme, S. P. 1998 'Environmental movements and democracy in Latin América' en Kelly, P. (ed.) Assessing democracy in Latin America (Boulder: Westview Press).

Hogenboom, B. 1998 Mexico and the NAFTA environment debate: The transnational politics of economic integration (Amsterdam: International Books).

Hogenboom, B.; Alfie Cohen, M. y Antal, E. 2003 'Crossborder Activism and Its Limits: Mexican Environmental 
Organizations and the United States' en Cuadernos $\operatorname{del} C E D L A, \mathrm{~N}^{\circ}$ 13: 100.

Keck, M. y Sikkink, K. 1998 Activists beyond borders: Advocacy networks in international politics (Ithaca: Cornell University Press).

Kurzinger, E. et al. 1991 Política ambiental en México. El papel de las organizaciones no gubernamentales (México: Fundación F. Ebert-Instituto Alemán de Desarrollo).

Leff, E. (ed.) 2001 Justicia ambiental: Construcción y defensa de los nuevos derechos ambientales, culturales, y colectivos en América Latina (México D. F.: PNUMA).

Léonard, E. y Foyer, J. 2011 De la integración nacional al desarrollo sustentable. Trayectoria nacional y producción local de la política rural en México (México: CEDRSSA / IRD) p. 466.

Lutz Ley, A. N. y Salazar Adams, A. 2011 "Medio ambiente y organizaciones de la sociedad civil: análisis de las redes civiles ambientalistas en Hermosillo, Sonora" en Región y sociedad, 23(51): 5-41 [ISSN: 1870-3925; en internet].

Martínez Alier, J. 2002 The environmentalism of the poor: A study of ecological conflicts and valuation (Cheltenham: Edward Elgar).

Melucci, A. 1999 Acción colectiva, vida cotidiana y democracia (México: El Colegio de México).

Mollard, E. y Lopez, E. 2006 "L'instrumentalisation des ONG au Mexique en situation indienne et non indienne" en Autrepart, 37: 129-146.

Pacheco-Vega, R. 2005 'Democracy by proxy: Environmental NGOs and policy change in Mexico' en Romero, A. y West, S. (eds.) Environmental issues in Latin America and the Caribbean (Dordrecht: Springer).

Pacheco-Vega, R. y Obdulia, V. L. 2003 “Cooperación internacional para la protección ambiental: la formación de coaliciones en perspectiva" en Foro Internacional, 43(2): 403-428.

Payá Porres, V. A. 1994 Laguna Verde: la violencia de la modernización. Actores y movimiento social (México, D. F.: Instituto Mora).

Petras, J. 1997 'NGOs and Imperialism' en Monthly Review, 49(7): 10-27.

Pleyers, G. 2010 Alter-Globalization becoming actors in a global age (Cambridge: Polity Press) Prefacio de A. Touraine.

Pleyers, G. 2011 "Le réinvestissement de l'espace local par les mouvements mexicains: refuge après les impasses politiques 
ou creuset d'une nouvelle culture politique?" en Cahiers des Amériques latines, 66: 39-56.

Pleyers, G.; Mestries, F. y Zermeño, S. (coord.) 2009 Los movimientos sociales. De lo local a lo global (Barcelona / México: Anthropos).

Simonian, L. 1999 La defensa de la tierra del jaguar: una historia de la conservación en México (México: CONABIO / INESEMARNAP).

Smith, J.; Chatfield, Ch. y Pagnucco, R. (comps.) 1998 Transnational social movements and Global politics: solidarity beyond the state (Syracuse: Syracuse University Press).

Stearns, L. B. y Almeida, P. 2004 'The formation of state actor-social movement coalitions and favorable policy outcomes' en Social Problems, 51(4): 478-504.

Tarrow, S. 1998 Power in movement: Social movements and contentious politics (Cambridge: Cambridge University Press) $2^{\circ}$ edición.

Tarrow, S. 2005 The new transnational activism (Nueva York: Cambridge University Press).

Toledo, V. M. 1983 "La otra guerra florida” en Nexos, 6. 15-24 septiembre.

Toledo, V. M. 1992 "Utopía y naturaleza: El nuevo movimiento ecológico de los campesinos e indígenas de América Latina” en Nueva Sociedad, 122: 72-85.

Toledo, V.M. 2000 La paz en Chiapas. Ecología, luchas indígenas y modernidad alternativa (México D. F.: Ediciones Quinto Sol).

Torres, B. 1997 'Transnational environmental NGOs: Linkages and impact on policy' en MacDonald, G. J.; Nielson, D. L. y Stern, M. A. (eds.) Latin America's environmental policy in international perspective (Boulder: Westview).

Touraine, A. 1981 The voice and the eye: An analysis of social movements (Cambridge: Cambridge University Press).

Umlas, E. 1998 'Environmental networking in Mexico: The Comité Nacional para la Defensa de los Chimalapas' en Latin American Research Review, 33(3): 161-189.

Velázquez García, M. A. 2010 “Los movimientos ambientales en México" en Bizberg, I. y Zapata, F. (eds.) Los Movimientos Sociales (México: El Colegio de México) pp. 275-328.

Velázquez, M. A. 2005 "Relaciones entre organizaciones y movimientos sociales. Redes y oportunidades políticas: los casos de la Red Nacional de Acción Ecologista (Argentina) 
y la Red Nacional de Derecho a la Información Ambiental (México)" en Región y Sociedad, XVII(33): 33-70.

Velázquez, M. 2008 "La construcción de un movimiento ambiental en México. El club de golf en Tepoztlán, Morelos" en Región y Sociedad, $X X(43)$ : 61-96.

Velázquez, M. A. 2009 Las luchas verdes. Los movimientos ambientalistas de Tepoztlán, Morelos y el Cytrar en Hermosillo (Hermosillo: El Colegio de Sonora).

Velázquez Guzmán, M. G. 1982 "Afectaciones petroleras en Tabasco: el movimiento del Pacto Ribereño" en Revista Mexicana de Sociología, 44(1): 167-187.

Verduzco Chávez, B. 2002 Conflictos ambientales. La internacionalización de la defensa de las comunidades contra instalaciones contaminantes (México: Universidad de Guadalajara).

\section{LISTADO DE SIGLAS}
ANAA
Asamblea Nacional de Afectados Ambientales
CCMSS
Consejo Civil Mexicano para la Silvicultura
Sostenible
CECCAM
Centro de Estudios para el Cambio en el Campo
Mexicano. Maderas del Pueblo del Sureste
CIEPAC Centro de Investigaciones Económicas y Políticas
Acción Comunitaria
CONCLAVE Coordinadora Nacional Contra Laguna Verde
ERA Estudios Rurales y Asesoría
FECOMEX Federación Conservacionista Mexicana
GIRA
Grupo Interdisciplinario de Tecnología Rural
Apropiada
PAIR Programa de Aprovechamiento Integral de Recur- sos Naturales
PSSM Proyecto Sierra de Santa Marta
RAFI Rural Advancement Foundation International, luego nombrada ETC Group, en 2001.

CÓMO CITAR ESTA PUBLICACIÓN:

Foyer, Jean and David Dumoulin Kervran. 2017. “Ambientalismo de las ONG versus ambientalismo de los pobres?” Pp. 393-414 en Paul Almeida y Allen Cordero Ulate, Eds., Movimientos Sociales en América Latina: Perspectivas, Tendencias y Casos. Buenos Aires: CLACSO. 